\title{
Kopulative Konjunktionen im Deutschen und Arabischen
}

\author{
Vorgelegt von: Hochschullehrerin
}

Afrah Majeed Hadi

DOI: $10.37648 / \mathrm{ijrssh.v10i04.019}$

Received:20 th September, 2020; Accepted:22nd October, 2020; Published:28th October, 2020

\section{EINLEITUNG}

Es gibt wirtschaftliche, politische und kulturelle Beziehungen zwischen Deutschland und der arabischen Welt, die sich seit dem Ende des Zweiten Weltkriegs entwickelt haben. Das Interesse an Fragen des Spracherwerbs und des Studiums der Übersetzung in sprachlichen Bereichen zeigt große Unterschiede zwischen den beiden Sprachen, da sie jeweils zu einer anderen Sprachfamilie gehören. Während die deutsche Sprache zur indogermanischen Sprachfamilie gehört, gehürt das Arabische zur semantisches Sprachfamilie.

Die Sprachliche Äußerung erfolgt in Sätzen. Jeder Satz ist aus einer oder mehreren kleineren Einheiten bestehende Redeeinheit. Diese Sprachlichen Einheiten werden durch Konjunktionen verbunden, die in zwei Gruppen eingeteilt werden, koordinierende und subordinierende Konjunktionen. Die vorliegende Arbeit beschäftigt sich mit kopulativen Konjunktionen im Deutschen und Arabischen und ist ein Versuch, die sprachlichen Ähnlichkeiten und Unterschiede zwischen den beiden Sprachen auszuführen und die kopulativen Konjunktionen im Deutschen und ihre entsprechende Übersetzungsmöglichketen im Arabischen anzuzeigen.

Die Uneinigkeit der deutschen Grammatiker über die Benennung von kopulativen Konjunktionen macht uns sorgfältig bei der Auswahl der geeigneten Gruppe für die Arbeit. Das Wort kopulativ bedeutet verbindend, verknüpfend und anreihend; Zur Untersuchung wird die Konjunktionen und, sowohl ..... als auch, nicht nur ..... sondern auch, sowie und weder ... noch , die von Gerhard Helbig und Joachim Buscha klassifiziert werden.

Der Vergleich ergibt hinsichtlich der kopulativen Konjunktionen große Unterschiede zwischen den beiden Sprachen, die dazu durch die Untersuchungsergebnisse beitragen, die Schwierigkeiten bei der Übersetzung zu überwinden.

\section{DIE THEORETISCHE GRUNDLAGE}

Konjunktionen ( lat. coniunctio,' Verbindung', 'Bindewort' - Auch: Bindewort, Subjunktion ) sind " Unflektierbare und nicht satzgliedfähige Wortart, deren Vertreter syntaktische Verbindungen zwischen Wörtern, Wortgruppen oder Sätzen herstellen und zugleich semantische Beziehungen zwischen diesen Elementen kennzeichnen. "1 Sie verbinden nicht nur Wortteile, sondern auch Gliedteile, Satzglieder und Sätze und drücken ein bestimmtes Verhältnis dieser Elemente zueinander aus:

-Vor- und Nachsilbe sind wichtig zu lernen. (Wortteile)

-Fleißige und faule Studenten bestehen die Prüfung. (Gliedteile)

-Er kauft einen Computer und moderne Ausstattung. (Satzglieder)

-Wir diskutieren drei Stunden, ohne dass wir eine Lösung finden. (Sätze)

${ }^{1}$ Vgl. Bußmann, Hadumond, 2002, S.364 
Man teilt die Konjunktionen in zwei Gruppen ein, koordinierende und subordinierende Konjunktionen. Der Unterschied zwischen diesen beiden Gruppen besteht darin, dass koordinierende (auch nebenordnende) Konjunktionen Sätze gleichen Grades verknüpfen, während subordinierende (auch unterordnende) Konjunktionen Sätze unterschiedlichen Grades verbinden. Daneben unterscheidet man Satzteilkonjunktionen, die keine Sätze verbinden, sodern Satzglieder oder Attribute, ${ }^{1}$ Helbig, Gerhard und Buscha, Joachim nennen diese kleine Gruppe, die nur den Konjunktionen als und wie gehören, "Adjunktionen". ${ }^{2}$ Im Unterschied dazu stehen andere Konjunktionen, die weder Wörter noch ${ }^{3}$ Wortgruppen verbinden kann, wie die Konjunktion "denn"; andere Konjunktionen dagegen wie "aber" kann keine Wortteile verknüpfen. ${ }^{4}$

Neben den Konjunktionen, die ein wichtiges Mittel der Verbindung von Satzelementen sind, gibt es noch weitere Ausdrucksformen, die ein formales Verknüpfungszeichen fehlt, die Asyndese, die Benennung bei der Einordnungsergänzung und die enge Apposition. In diesem Fall stehen die Wörter mit Aneinanderreihung gleicher Elemente:

-alte verfallende Häuser (Asyndese)

-Wir nennen sie eine Lügnerin. (Einordnungsergänzung)

-Herr Robert, Frau Petra (Apposition)

Hinsichtlich ihrer semantischen Beziehungen lassen sich die koordinierenden Konjunktionen in verschiedene Gruppen eingeteilt: kopulativ, kausal, alternativ, adversativ, restriktiv und konkretisierend, während die subordinierenden Konjunktionen wegen ihrer Verwendung unterschiedliche Gruppen kennzeichnen: kausal, konsekutiv, konditional, konzessiv, modal, temporal, vergleichend, restriktiv, adversativ, final und für Ersatzhandlungen. ${ }^{5}$

\subsection{Der Begriff Konjunktion}

Unter dem Begriff Konjunktion versteht man „unflektierbare und nicht satzgliedfähige Wortart, deren Vertreter syntaktische Verbindungen zwischen Wörtern, Wortgruppen oder Sätzen herstellen und zugleich semantische Beziehungen zwischen diesen Elementen kennzeichnen."6 In der deutschen Sprache sind verschiedene Begriffe und verschiedene Oberbegriffe von Konjunktionen vorhanden. Eisenberg unterteilt die Konjunktionen in zwei Gruppen. nämlich koordinierende- und subordinierende Konjunktionen, die Konjunktoren und Subjunktoren benannt werden. Ulrich Engel ist die im weitern Sinn nichtflektierbare gezählte Partikeln als Oberbegriff zu betrachten. Gerharg Helbig und Jochim Buscha unterscheiden zwischen Konjunktionen und Subjunktionen. Bei Autoren von Duden wird der Oberbegriff Junktion in Konjunktion und Subjunktion unterteilt. Koordinierende- und Subordinierende Konjunktionen werden bei Zifonun und Engel als Konjunktoren und Subjunktoren klassifiziert, während diese bei Helbig/ Buscha und Duden - Grammatuk als Konjunktionen und Subjunktionen genannt werden. ${ }^{7}$ In dieser Arbeit wird die Bezeichnung vom Begriff Konjunktionen für die behandelten kopulativen Konjunktionen übernommen.

\section{KOPULATIVE KONJUNKTIONEN IM DEUTSCHEN}

Das Wort kopulativ steht im Sinn verbindend, verknüpfend und anreihend. ${ }^{8}$ Dazu gehören eine Gruppe von Konjunktionen, die sowohl Sätze als auch Teilsätze oder kleinere Einheiten aneinander gereiht werden. In dieser Gruppe gehört die Konjunktion und, die als die häufigste Konjunktion der deutschen Sprache zu betrachten ist. Unter kopulativen

\footnotetext{
${ }^{1}$ Vgl.Götze, Lutz/ Hess- Lüttich, Ernest W. B., 2002, S.315 - 319

${ }^{2}$ Vgl. Helbig, Gerhard und Buscha, Joachim: Deutsche Grammatik, S.416

${ }^{4}$ Vgl.Hoberg, Ursula/ Rudolf, 1979, S.315

${ }^{4}$ Vgl.Götze, Lutz/ Hess- Lüttich, Ernest W. B., 2002, S.315 - 320

Vgl. Götze, Lutz/ Hess, Ernest W. B., S. $316-319^{5}$

Vgl. Bußmann, Hadumod, S. $364^{6}$

Vgl. Hraishawi, Maher, S. $31-32^{7}$

Vgl.Wahrig - Deutsches Wörterbuch, 2002, S.880 ${ }^{8}$
} 

manchmal von einem Grammatiker zum anderen unterschiedlich sind. Gerhard Helbig und Joachim Buscha klassifizieren die Konjunktionen Und, sowohl .... als auch, nicht nur .... sondern auch, sowie und weder ... noch unter dem Begriff kopulativen Konjunktionsn. ${ }^{1}$ Bei Ulrich Engel ist nicht nur ..... sondern auch nicht unter kopulative Konjunktionen zu betrachten; er klassifiziert sie unter adversativen Konjunktorn, weil sie einen Gegensatz signalisiert. ${ }^{2}$ Lutz Götze und Ernest W. B. Hess- Lüttich klassifiziert weder ... noch jedoch unter alternativen Konjunktionen. ${ }^{3}$ Die Autorn von Duden Grammatik Klassifiziern jedoch darunter die Konjunktionen und, sowie, plus und wie, sowohl .... als (auch)/ wie (auch), weder .... noch. ${ }^{4}$

\subsection{Morphologischer und syntaktischer Hinsicht}

Nach morphologischer Hinsicht gehören die kopulativen Konjunktionen im wesentlichen zwei Klassen an:

1. Einteilige einfache Konjunktionen: und, sowie (keine Formvarianten).

2. Mehrteilige korrespondierende Konjunktionen: weder .... noch, nicht nur ... sondern auch, sowohl .... als auch/wie auch (mehrere Formvarianten). Sie verbinden gleichgeordnete Elememte miteinander ohne Änderung sowohl die Stellung des Verbs als auch die Abfolge der Satzglieder.

Die Konjunktionen können nach syntaktischen und nach semantischen Gesichtpunkten eingeteilt werden.

Die Aufgabe der kopulativen Konjunktionen besteht darin, syntaktische Elementen zu koordinieren. In semantischer Hinsicht dagegen gibt es weniger Möglichkeiten als bei den subordinierenden Konjunktionen, außerdem ist der Inhalt mehr theoretisch.

Und, sowohl .... als auch, nicht nur .... sondern auch, sowie und weder ... noch sind in deutschen Grammatiken als koordinierende Konjunktionen klassifiziert. Wenn ein Subjekt der 1. und ein Subjekt der 2. Person durch diese Konjunktionen miteinander verbunden, dann richtet sich das finite Verb nach der 1. Person Plural; durch wir können die beiden Subjekte zusammengefasst werden: ${ }^{5}$

-Du und ich (= wir) arbeiten in diesem Büro.

-Weder ich noch du (= wir) sind in diesem Jahr gefahren.

-Sowohl wir als auch sie (= wir) haben den Weg gefunden.

Bei hauptsatzverbindenden Konjunktionen steht das Verb hinter der Konjunktion:

-Sie fährt um 8 Uhr und sie wird morgen nachkommen.

Es ist ersichtlich, dass Verben der beiden Hauptsätze in Person und Numerus übereinstimmen. Daneben verknüpfen die anreihenden Konjunktionen Satzglieder, die derselben Satzgliedklasse angehören müssen, z.B. zwei Subjekte, zwei Nominalergänzungen .... usw.; man kann demnach nicht eine Lokal- mit einer Modalangabe durch kopulative Konjunktionen verbinden, Sie müssen gleichartig und gleichrangig sein: ${ }^{6}$

-Weder die Mutter noch der Vater haben ihn gesehen.

Vgl. Helbig, Gerhard/ Buscha, Joachim: Deutsche Grammatik, S $395^{1}$

Vgl. Engel, Ulrich, S. $740^{2}$

Vgl. Götze, Lutz/ Hess- Lüttich, Ernest W. B., S.315 ${ }^{3}$

Vgl. Autorenkollektiv: Duden, 2006, S. $629{ }^{4}$

Vgl. Vgl. Helbig, Gerhard/ Buscha, Joachim, S. $536^{5}$

Vgl.Engel, Ulrich: Deutsche Grammatik, 1996, S.738 ${ }^{6}$ 
-Wir möchten weder nach Berlin noch nach München fahren.

Daneben kann weder... noch ganze Sätze miteinander verknüpfen. In diesem Fall steht der erste Teil der Konjunktion frei beweglich, d.h. dass er sich syntaktisch wie ein Adverb verhält, der zweite Teil dagegen muss immer am Beginn des zweiten Verknüpfungselementes sein:

-Weder braucht er Geld, noch bittet er um Hilfe.

-Er braucht weder Geld, noch bittet er um Hilfe.

Im Gegenteil dazu sind die Konjunktionen sowie und sowohl als (auch) die einzigen, die nur Satzglieder und andere Wortgruppen oder Wörter verbinden können:

-Gern möchte ich alle europäischen Sprachen sowie Persisch lernen.

\subsection{Bedeutung und Gebrauch im Einzelnen}

\subsection{1 und}

Das Bindewort und ist der häufigst gebrauchte aller Konjunktionen und gilt als Standardfall der koordinativen Fähigkeiten überhaupt, denn es steht zwischen den gehäuften Elementen. Mit und können Morpheme, Wörter, Wortgruppen, Phrasen, kommunikative Minimaleinheiten und Sätze koordiniert werden. Es hat keine Heteroseme in anderen Wortarten und keine Varianten in einer anderen syntaktischen Konnektorklasse.

Der Ausdruck der natürlichen Sprache und die logische Konjunktion stimmen in ihrer Bedeutung nicht vollständig überein, da die Verwendung von und z.B. sekundäre Bedeutungen kausaler Natur vorschlagen kann. Petra kommt und Martin geht wird nicht einfach bedeuten: Es kommt zum Vorschein zwei Sachverhalte, die beide wahr sind, „1.Petra kommt“, „2.Martin geht“. Im Folgenden wird nur die natürliche Sprache, nicht der logische Ausdruck berücksichtigt.

Normalerweise nimmt man die gleichen Bestandteile an und hat die Funktion, sie zu einem anderen Bestandteil zu kombinieren, der im Satz die gleiche Rolle spielt wie die einzelnen Bestandteile. Diese Annahme gilt jedoch mit und nicht vollständig koordiniert.

Erstens kommt es Fälle vor, wie der große und der kleine Peter, Eva kocht Bohnensuppe und Hans Kartoffeln sind sicherlich keine Bestandteile, hier werden die Rückstände der Bestandteile koordiniert.

Zweitens kann mit und mehrfach koordiniert werden. Dann ist jedoch nicht jedes Paar mit einem separaten Bestandteil verbunden. Die weniger Komplexe 2. Struktur in der folgenden Abbildung beschreibt die Situation angemessen.

Der Konjunktion und enthält weder Heterogenität in anderen Wortarten noch andere Variablen in der Kategorie der grammatikalischen Dirigenten.

Und ist dagegen relativ ausgeprägt: Es kann symbolisch für zeitlich allmälich eintretende, und-dann und konsekutive und deshalb andere Interpretationen sein, bei denen der Austausch von Bindungen bedeutungslos ist.

- Ein Schuss fiel und ein Mädchen schrie auf.

- $\neq$ Eine Frau schrie auf und ein Schuss fiel.

Im Rahmen einer umfassenden Forschung wird allgemein die Arbeit der strukturellen Ikonographie angenommen.

- Ein Schuss fiel und ein Mädchen schrie, aber die Glaube ist, der Schrei kam vor dem Schuss.

- A: Stimmt es, dass ein Mädchen geschrieen hat und ein Schuss fiel?

B: Ja, beide sind richtig: Ein Schuss fiel und ein Mädchen schrie auf.

Ja, beide sind richtig: Ein Mädchen schrie auf und ein Schuss fiel. ${ }^{1}$ 
Und - die Koordinaten werden normalerweise als prägnant formatierte Sätze interpretiert, in denen alle identischen Teile in den beiden koordinierten Sätzen weggelassen werden, zum Beispiel: Boris nimmt Valium und Verona Librium. Es kann zurückverfolgt werden: Boris nimmt Valium und Verona nimmt Librium. Es stellt sich jedoch heraus, dass nicht jede Koordination auf diese Weise erklärt werden kann: Wolfgang und Helmut verlassen das Parlament bedeutet nicht Wolfgang verlässt das Parlament und Helmut verlässt das Parlament . Kongruenzphänomene sollten auch erklärt werden, wenn man von den Überresten harmonisierter Sätze ausgeht, auch wenn ein gemeinsames Verfahren nicht beschrieben wird. ${ }^{1}$

Bei wiederholter Koordination steht und nur zwischen allen Konjunktionen, wenn die Verknüpfung auf diese Weise besonders wahrscheinlich sein soll: Wir verkrochen und träumten und dachten an Selbstmord. Dies hat eine größere Trennwirkung für verbundene Elemente. Andernfalls wird der zweite durch Nebeneinander (gekennzeichnet durch ein Komma oder eine kurze Pause und eine fortschreitende Intonation) mit dem vorletzten des Arbeitszyklus verbunden, wobei der letzte ein Arbeitszyklus ist: Wir verkrochen uns träumen, dachten an Selbstmord. Wenn Ausdrücke in der StandardSingularform formatiert sind, liegt das finite Verb im Plural vor: Eva und ihre Tochter konnten so nicht weiterarbeiten. Die Funktion von und liegt in der Herstellung von funktionaler Konvergenz allgemeiner Art: der Subsumierung im Rahmen einer gemeinsamen Funktion, der Integration von Sachverhalten in eine Reihe von Sachverhaltenskomplex, Verknüpfung sprachlicher Handlungen mit einem Muster oder Musterkomplex, Zusammenbindung von Gegenständen, Charakteristika oder Modifikationen. Der Zusammenstoß von Konjunkten induziert - abhängig von der damit verbundenen Bedeutung und dem Kontext - eine spezifische Relationierung, ohne dass diese Beziehung durch einen passenden Konnektor (Konjunktor, Adverb, Partikel) gekennzeichnet sein müßte. Auf die Herstellung der grundlegenden Konvergenz ist die Zuständigkeit von und beschränkt. ${ }^{2}$

Ulrich Engel behandelt in Bezug auf die Verwendungsweise die Bedeutung der Konjunktor und; er meint, dass diese fünf verschiedene Bedeutungen hat, gemeinsame Geltung, Bedingung, gegensätzliche Sachverhalte, unbegrenzte Steigerung und in einer letzten Verwendungsweise handelt es sich um Verbindung zwei Sätze; Während der erste die Funktion den Ausdruck der Höflichkeit oder der Modlfikation des Hauptgeschehens hat, wiedergibt der zweite den wesentlichen Inhalt.

1- Gemeinsame Geltung zweier gehäufter Elemente signalisiert die Konjunktion und:

-Wir wünschen ihm viel Glück im Beruf und im Privaten Leben.

-Sie verlor Geld und Vertrauen.

Diese Äußerungen zeigen, dass und Elemente beliebigen Umfangs und beliebigen grammatikalischen Ranges kombinieren; Die einzige Bedingung bleibt, dass die gehäuften Elemente gleichartig und gleichrangig sind.

Wenn und die gemeinsame Geltung bezeichnet, konkurriert es auch mit sowie und mit sowohl .... als (auch). Es ist mit und stilistisch neutral und kann daher in beliebigen Textsorten verwendet werden, während die konkurrierenden Konjunktionen stilistisch verfeinert sind und hauptsächlich in der geschriebenen Sprache vorkommen. Ein weiterer Unterschied besteht darin, dass die Konjunktion und die gemeinsame Gültigkeit der gehäuften Elemente angibt, die neutral gestapelt sind, während diese Eigenschaft durch sowie beim zweiten Element, durch sowohl .... als (auch) bei beiden Elementen hervorgehoben wird. Gelegentlich kann und am Satzanfang stehen. Der Punkt, der den vorherigen Satz abschließt, kann darauf hinweisen, dass und hier nicht zwei gleichrangige und gleichartige Elemente miteinander verbunden sind. Aber in solchen Fällen - Hugo lachte laut, alle drehten sich zu ihm um. Und niemand bemerkte, dass Daniela das Bücherregal manipulierte. Die Join Connect-Funktion ist stärker als der Punkt, sodass die Definition erfüllt ist. Auch wenn es sich um Textabschnitte, Kapitel, ganze Romane oder sogar einen neuartigen Titel handelt - siehe. Und führen Sie dorthin, wo Sie nicht wollen ... um zu beginnen, und es liegt kein Verstoß gegen die angegebene Definition vor: Der Autor möchte solche Formeln verwenden, um die Hinzufügung des vorherigen Satzes zu erreichen. Auf diese Weise lässt er den Hörer sich eine imaginäre Welt vorstellen, auch wenn nicht jedes Element dieser Welt sprachlich wahrgenommen wird:

\footnotetext{
${ }^{1}$ Vgl. Albert, Ruth, S.146

Vgl. Zifonun, Gisela/ Hoffmann, Ludger und andere, S. $2391^{2}$
} 
-,, Hugo lachte laut auf, alle wandten sich ihm zu. Und niemand bemerkte, daß sich Daniela derweil am Biücherschrank zu schaffen mach te. "1

Die Verbindungsfunktion der Konjunktion und ist aber in solchen Fällen stärker als der Punkt, damit ist die Definition erfüllt. Durch solche Formulierungen wird ein Vorgängersatz hinzugedacht, und auf diese Weise vermittelt es dem Hörer den Eindrück einer fiktiven Welt, auch wenn nicht jedes Element dieser Welt in der Sprache realisiert ist.

2-Konditional ist die zweite Bedeutung von der Konunktion und, die Sätze verknüpft, von denen der erste Satz die Bedingung für das Geschehen des zweiten Satzes nennt. Das finite Verb des ersten Satzes ist oft im Imperativ:

-Geh nach links und du stehst vor dem Bahnhof!

Solche Satzfolgen entsprechen Konditionalgefügen wie:

-Wenn du nach links gehst, (dann) stehst du vor dem Bahnhof!

Im Vergleich zu solchen normalen konditionalen Satzgefüge sind die genannten durch und Satzkombinationen lebindiger und unmittelbarer. Und der Schreiner kann, wenn er im bedingten Sinne verwendet wird, nicht durch die Kopplung ersetzt werden und auch ... wie (auch) (aber gegen das Unterbewusstsein, wenn).

Bei der konditionaler Bedeutung ist die Konjunktion und nicht gegen die zweigliedrigen Konjunktoren sowie, sowohl .... als (auch) austauschbar.

3-Adversativ: Die Konjunktion und verbindet Sätze, die gegensätzliche Sachverhalte beschreiben:

-Die Wohnung ist ideal und unbezahlbar.

Adversatives und hat eine zusätzliche Funktion, dass diese Art auch Verwunderung, Verärgerung und Empörung ausdrücken kann.

4-Graduativ: Durch Verbindung gleichlautender Komparativformen eines Adjektivs kann und eine unbegrenzte Steigerung ausdrücken:

-Der Krach wurde lauter und lauter.

Diese Form kann durch die Wortgruppe immer + Adjektiv im Komparativ ersetzt werden:

-Der Krach wurde immer lauter.

Diese Formulierung ist stilistisch neutral, während die und- Konstruktion intensiver ist, und anzeigt, dass eine bestehende Erwartung oder eine geltende Norm überschritten wurde.

5-In einer letzten Verwendungsweise kann und darüber hinaus zwei Sätze in Verbindung stehen. Der erste Satz dient nicht nur dem Ausdruck der Höflichkeit sondern auch der Modifikation des Hauptgeschehen durch Formulierung eines Adverbs oder einer sonstigen Angabe, der zweite dagegen gibt den wesentlichen Inhalt wieder ${ }^{2}$ :

-Sei so gut und schick mir mal Geld.

-Schick mir bitte mal Geld.

Gisela Zifnum, Ludger Hoffmann und andere behandeln dabei vielfältige Möglichkeiten der Verwendungsweise der Konjunktion und, wie temporal, lokal, alternativ, inkrementiv, kausal, konsekutiv, konzessiv, instrumental, komitativ und restriktiv. $^{3}$

Vgl. Engel, Ulrich, S. $746^{1}$

${ }^{2}$ Vgl. Engel, Ulrich: Deutsche Grammatik, 1996, S. 746 - 747

Vgl. ${ }^{2}$ Breindl, Eva: Handbuch der deutschen Konnektoren, 2006, S.13

Vgl. Zifonun, Gisela/ Hoffmann, Ludger und andere, S. $2393-2394^{3}$ 


\subsection{2 sowie}

Die Konjunktion sowie zeigt gemeinsame Geltung an; diese Eigenschaft wird im zweiten Element eigens hervorgehoben: -Ihre Aufgabe ist es, die Adressen ab-sowie umzuschreiben.

Mit sowie sind keine Verbgruppen, Haupt- und Nebensätze oder kommunikativen Minimaleinheiten koordinierbar. Die Funktion von sowie, die nur kleinere Elemente verbinden kann, besteht darin, eine nachträgliche und oft nachrangige Erweiterung durchzuführen:

-Peter und Eva trugen eine Kiste die Treppe hinauf.

-Peter sowie Eva trugen eine Kiste die Treppe hinauf.

Was über Element mit und gesagt wurde, gilt auch für Element mit sowie. Somit betont der hinweisende Teil das Element so, auf das sich das zweite Element unter seinem Funktionsaspekt bezieht, nicht unbedingt von gleicher Bedeutung, während wie Vergleichbarkeit ausdrückt (etwa hinsichtlich einer Ereignisbeteiligung). Daraus folgt, dass sowie keine bloße Variante von und ist. Vor allem wird es nicht verwendet, wenn zwei Elemente auf dieselbe und untrennbare Weise an einem Sachverhalt partizipieren. Für die Äußerung mit sowie wäre also die bevorzugte Lesart, dass Peter und Eva eine Kiste getrennt (nacheinander) hinaufgetragen haben. Wie kombiniert gleichrangige Elemente unter dem Aspekt ihrer Vergleichbarkeit, mit sowie dagegen wird das Folgekonjunkt in eine Reihe mit dem ersten gestellt, wodurch so gerade die Gleichwertigkeit eines Gehalts hervorgehoben wird. ${ }^{1}$

In beschränktem Umfang ist sowie gegen die Konjunktionen und und sowohl .... als auch austauchbar.

Sowie kann auch als Subjunktion unmittelbarer Aufeinanderfolge gebraucht werden, es betrifft nicht die Verbindung von Sätzen gleichen Grades: ${ }^{2}$

-Sowie ich in Bagdad angekommen bin, rufe ich dich an.

3.2.3 sowohl .... als (auch), sowohl .... wie (auch)

Die Doppelkonjunktionen sowohl ... als auch, sowohl ... wie auch charakterisieren als getrennt, mehrteilig und kopulativ. Sie signalisieren auch gemeinsame Geltung, wobei diese Besonderheit bei beiden gehäuften Elementen in gleicher Weise festgestellt wird:

-Sie ist sowohl Zahnärztin als auch (wie auch) Künstlerin.

Diese beiden Doppelkonjunktionen verknüpfen weder Hauptsätze noch Nebensätze, sondern nur Teile von solchen, wie Morpheme, Wörter, Phrasen und Verbgruppen; damit können auch Sätze, nicht aber kommunikative Minimaleinheiten koordiniert werden. Während der erste Teil jeweils vor dem ersten gehäuften Element steht, steht der zweite Teil vor dem zweiten gehäften Element.

Im gewissen Umfang sind die beiden Doppelkonjunktionen sowie und und austauschbar. ${ }^{3}$ Neben der gleichen Geltung beider Doppelkonjunktionen wird semantisch auch die Gleichgewichtigkeit hervorgehoben. Bei Verneinung steht für sowohl .... als auch die Konjunktion weder ... noch.

\subsection{4 weder .... noch}

Die Konjunktion weder ... noch negiert die gehäuften Elemente und stellt dabei fest, dass die Negation für beide in gleicher Weise gültig ist. Weder ... noch verbindet nie Sätze, sondern nur kleinere Elemente. ${ }^{4}$ Morphem-, Wort-, Phrasen-,

Vgl. Zifonun, Gisela/ Hoffmann, Ludger und andere, S. $2396{ }^{11}$

Vgl. Helbig, Gerhard/ Buscha, Joachim, S. $602^{2}$

Vgl. Engel, Ulrich, S. $745^{3}$

Vgl. Ebenda, S.758 ${ }^{4}$ 
Verbgruppen-, Satz-, und KM- Konjunkte werden mit dem Universalkonjunktion weder .... noch verbunden. In der Regel gibt es zwei Arten, aber je nach den Beispielen können auch mehr mit dem zweiten Teil verbunden werden:

-,Weder verwechselt sie die Welt, die sie als Ihresgleichen nennt, mit sich, noch sind ihre Kategorien losgelassene des bloßen Subjekts: zugeeignet bleiben sie die der Welt.

-,,Ich habe weder Magenschmerzen noch schlaflose Nächte noch Herzflat- tern. “I

Die Verfahren der Analepse und Katalepse können verwendet werden. Eine Assertion-Zeichenfolge mit so verbundenen Konjunkten auf diese Weise ist nur gültig, wenn die Teil- Assertionen falsch sind. Sämtliche Konjunktionsteile wirken sich negativ aus.

\subsection{5 nicht nur ...... sondern auch}

Die getrennte mehrteilige Konjunktion nicht nur .... sondern (auch) bezeichnet eine Hinzufügung einen hervorgehobenen Zusatz. Diese verbindet nur kleinere Elemente. Dabei wird die Exklusivität des ersten Elements der gehäuften Elemente aufgehoben und als Bestätigung die Gültigkeit des zweiten Elements hervorgehoben:

-Nicht nur Eva, sondern auch Peter ist gekommen.

Wenn auch Sätze verbunden werden, dann müsste der erste Teil (nicht nur) zu den Rangierpartikeln gezählt werden, denn er ändert die Wortreihenfolge:

-Nicht nur hat Eva ebenfalls mitgearbeitet, sondern Peter hat auch Geld gespendet.

Gleichbedeutend ist eine Konstruktion, die den ersten Satz in die Sequenz (als Nebensatz) mit dass einleitet, reproduziert jedoch den zweiten Satz ohne sondern, aber in der Regel mit auch:

-Nicht nur dass Eva ebenfalls mitgearbeitet hat, auch Prter hat Geld gespendet. ${ }^{2}$

Außerdem kann der zweite Teil weggelassen oder durch Juxtaposition ersetzt werden:

-, Nicht nur die Stadt und das Interieur [ ], die Stadt und das Freie vermö- gen sich zu verschränken.“3

\section{KOPULATIVE KONJUNKTIONEN IM ARABISCHEN}

Die arabische Sprache ist eine der wichtigsten Sprachen, die auf der ganzen Welt verbreitet sind. Sie wird von mehr als zwei Milliarden Menschen auf der Erdoberfläche gesprochen. Daher ist es unerlässlich, sie gut zu studieren, ihre Eigenschaften kennenzulernen und in ihre Wissenschaften einzutauchen, um sie richtig zu lernen. Im Arabischen unterscheidet man zahlreiche detaillierte Konjunktionen, die Wörter, Phrasen, Sätze oder Satzteile miteinander verbinden, um eine bestimmte Beziehung zwischen diesen Teilen auszudrücken. Es gibt viele Verwendungsmöglichkeiten der areibischen Bindewörter, wie eine absolute Kombination von Sätzen, Ursache und Grund, eine Folge, konzessive Bedeutung, gleichzeitige Temporalsätze und Hinzufügung von Informationen. ${ }^{4}$ In dieser Arbeit wird auf die Verbindungsmittel أدوات الربط im Arabischen eingegangen, die nur den Kopulativen Konjunktionen im Deutschen entsprechen.

Vgl. Zifonun, Gisela/ Hoffmann, Ludger und andere, S. $2399^{1}$

Vgl. Engel, Ulrich, S. $745^{2}$

Vgl. Zifonun, Gisela/ Hoffmann, Ludger und andere, S. $2420^{3}$

Vgl. https://mawdoo3.com ${ }^{4}$ 
a-Und

Im Arabischen ist und الو اوف العطف 30 او und wird verwendet, mehrere Elemente in einem einfachen Satz zusammenzufassen. Diese Konjunktion bezeichnet im Arabischen eine absolute Kombination:

\section{-Mohammed und Khalil nahmen teil.}

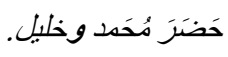

Diese Äußerung steht nicht im Sinn, dass Mohammed vor Khalil anwesend war, vielleicht hat Mohammed vor ihm teilgenommen, da es möglich ist, dass beide zusammen anwesend waren. Daher findet sich im heiligen Koran Unterschiede in Bezug auf Wortreihenfolge:

-,,Das diesseitige Leben ist nur Spiel und Zersteuerung. “ Koran 47/ 36

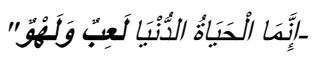

-,,Dieses irdische Leben ist nur Zersteuerung und Spiel. “ Koran 29/ 64

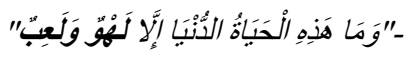

Das soll aber nicht heißen, dass es nicht die Anordnung angibt; vielmehr kann es in einer Reihenfolge und in anderen je nach Kontext vorkommen. Die Reihenfolge der einzelnen Glieder eines syntaktischen Ausdruck hängt aber von ihrer Wichtigkeit in einem Text, um darauf die Informationsempfänger aufmerksamkeit zu machen. ${ }^{31}$

Die Übereinstimmung der beiden Sätze in den Nachrichten oder in der Konstruktion erfordert die Verbindung zwischen ihnen, wenn der Anlass oder das Ganze die Verbindung rechtfertigt; dementsprechend nimmt die Bedeutung der Verbindung mit und an Stärke und Sichtbarkeit zu, und die Angelegenheit dann explizit. ${ }^{32}$

Außerdem können sich das Prädikat und das Subjekt vereinigen, und sie finden keine Rechtfertigung für die Verbindung:

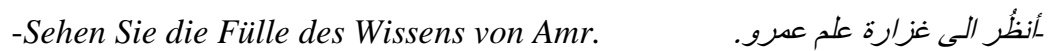

Diese Äußerung wird trotz der Vereinigung des Prädikats und des Subjekts nicht in einem einzigen Kontext kombiniert, weder getrennt noch verbunden.

Möglicherweise kann jede von ihnen in den beiden Sätzen unterscheiden, es liegt eine Begründung für die Verbindung vor:

-,,Als sie (wieder) bei ihm eintraten, sagten sie: „, O hoher Herr, Unheil ist uns und unseren Angehörigen widerfahren. Und wir haben (nur) Ware von geringem Wert gebracht. “ Koran 12/88

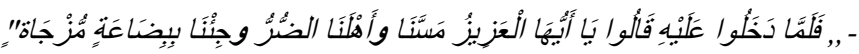

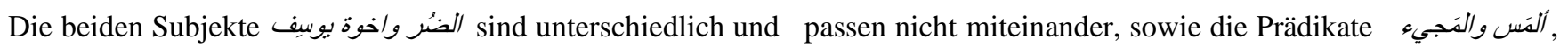
Trotzdem werden die beiden Sätzen verbunden, weil es eine Rechtfertigung für die Verbindung gibt, nämlich dass Grund für das ألمَجِيءist.

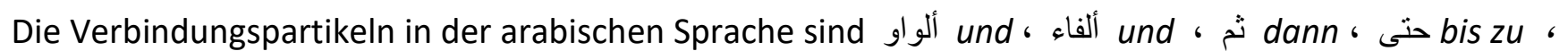

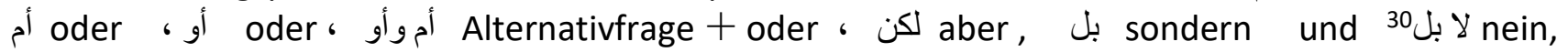
aber. 
In der arabischen Sprache kann الواو zwischen dem Adjektiv الصال und seinem abhängigen Wort und zwischen dem Akkusativ $^{33}$ und dem Wort, das damit zusammenhängt, ob sowohl الصفة als auch الحال ein Wort oder ein Satz ist. ${ }^{34}$

Die arabischen Grammatiker erwähnen, dass الواو durch idividuelle Bestimmungen (أحكام الواو ) gekennzeichnet ist:35

1-InVerbindung mit إمّا 1.

-Entweder hat er unsere Verabredung vergessen, oder er hat nicht kommem können. إما انه نَسي موعنا (أو) وإما لم بيتطع الحضور

Eine der auffälligsten Methoden im heiligen Koran besteht darin, die Aspekte und Möglichkeiten in einem Fall unter Verwendung von entweder .... oder und anderen Sprachlichen Methoden zu überprüfen: ${ }^{36}$

-,, Und (wieder) andere werden zurückgestellt, bis die Anordnung Allahs (ergeht). Entweder straft Er sie, oder Er nimmt ihre Reue an. Allah ist Allwissend und Allweise. “ Koran 9/ 106

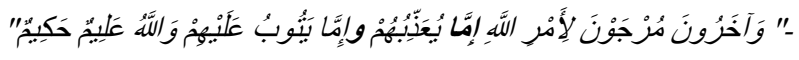

2-In Verbindung mit كن :

-Er kommt nicht zur Vorlesung, aber zu allen Feiern.

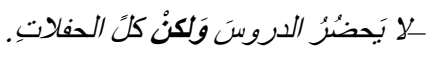

3-In Verbindung mit $\vee$, wenn ihm eine Verneinung vorausgeht. In diesem Fall nimmt durch eine andere Partikel ausgedrückte Verneinung wieder auf, falls kein neues Verb auftritt: ${ }^{37}$

-Er hat nicht das Bier und nicht den Wein getrunken. (Er hat weder das Bier noch den Wein getrunken.)

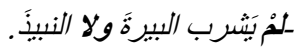

4- Bei Kardinal- und Ordinalzahlen

Die in denen Einer auftretenden Ordinalzahlen bestehen aus einer Kardinalzahl für die Zehnerzahlen und einer Ordinalzahl für die Einerzahlen. Dabei ist die abweichende Umsetzung der 2. In Ordinalzahlen wie 22., 32. Zu beachten: ${ }^{38}$

- der 22. Tag

$$
\text { - اليوم الثاني والثلاثون }
$$

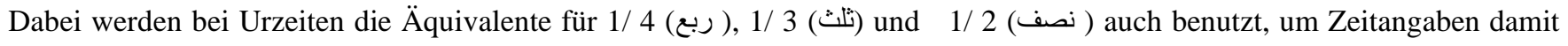
auszudrücken:

-um 2.30 Uhr

$$
\text { ـفي الساعة الثانية والنصف }
$$

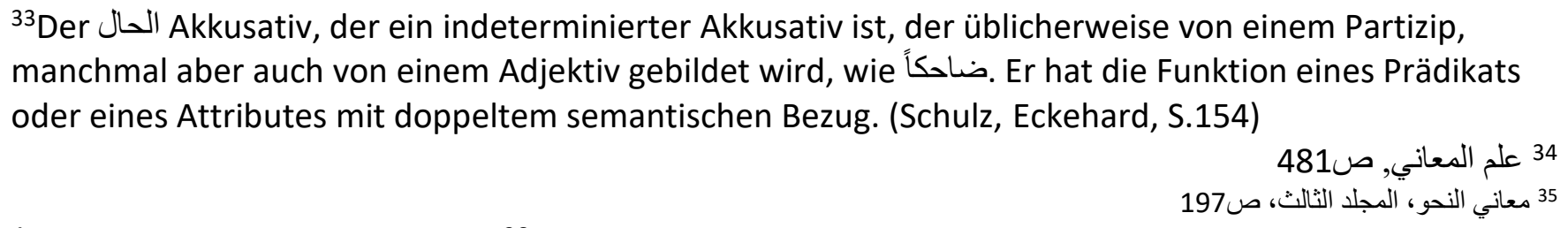

/https://qurblog.wordpress.com ${ }^{36}$

Vgl. Schulz, Eckehard, S.165 ${ }^{37}$

Vgl. Schulz, Eckehard, S.224 ${ }^{38}$ 
Um eine Begleitung mit zu bezeichnen tritt der Akkusativ nach der Verbindungspartikel und ein, besonders in Verbalsätzen und manchmal auch ohne Verbum:

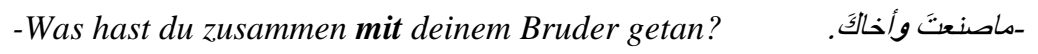

-Was hast du mit Amr (zu tun)?

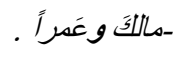

Daneben kann die Verbindungspartikel und als Schwurpartikel benutzt werden: ${ }^{39}$

-Bei Gott, das Leben ist schön.

واللهِ ، ألحياةُ جَمبلةه"

Die zweite Verbindungspartikel ist ألفاء und, die eine koordinierende und manchmal auch subordinierende Partikel zu betrachten ist. Sie wird vor allem dazu genutzt zwei oder mehr (Verbal)-Handlungen voneinander abzugrenzen. Sie kann temporale, konsekutive oder kausative oder adversative Bedeutung haben. Sie tritt oft in Bedingungs- und Konzessivsätzen auf und trennt dort die beiden Teile solcher Sätze. Es kommt häufig in Bedingungs- und Konzessivsätzen vor, da die beiden Teile dieser Klauseln getrennt sind $:^{40}$

-Die Delegationen und der Präsident kamen an.

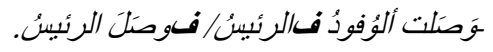

-Ich betrat das Haus und fand ihn in seinem Büro schlafend..

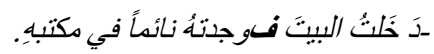

Die Verbindungspartikel $\mathbf{i}$ wird auch in Kolloktionen benutzt:

-Schneller und schneller.

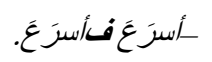

-Stück für Stück, eins nach dem anderen.

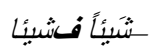

Das soll aber nicht heißen, dass Sätze mit der Verbindungspartikel فimmer der Konjunktion und im Deutschen entsprechen.

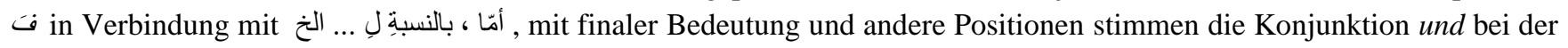
Übersetzung ins Deutsche nicht überein:

-Was den Minister betrifft, der ist in London.

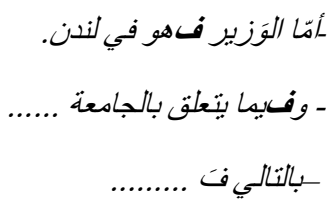

-Folglich.........

$\underline{\text { b- Sowie }}$

Die mehrteilige Konjunktion sowohl ..... als (wie) auch und sowie sind nachdrücklicher als und. Damit wird besonders betont, dass noch etwas hinzukommt:

-Er hat Romane und Novellen sowie einige Kurzgeschichte geschrieben.

كتب روايات وحكايات خر/فية وبعض الفصص القصيرة.

-Sowohl mein Vater als auch meine Mutter kommen aus dem Irak.

Vgl. Brockelmann, Carl, S.115, $148^{39}$

Vgl. Schulz, Eckehard, S.111 40 
أبي و أمي من العراق.

Die arabischen Partikeln أيضاً ، وكنلك ايضاً können neben الواو als Äquivalente für die deutsche Konjunktion sowie betrachtet werden.

c-Sowohl .... als auch

Außer und hat Im Arabischen die mehrteilige Verbindungspartikel sowohl ... als auch noch andere Übersetzungsmöglichkeiten, wie wَواء . أم

und

-Alle Länder, sowohl arabische als (wie) auch ausländische, haben unter Covid 19 gelitten.

ـكل الدول سواء العربية أم (أو) الأجنبية عانوا من كوفبي 19.

-Sowohl in Osteuropa als auch in Westeuropa ist die Kartoffel ein wichtiges Nahringsmittel.

ـالبطاطس من اهم المواد الغذائية في كل من اوربا الثرقية واوربا الغربية.

-Sowohl er als auch sie.

- هو وهي معاً.

-,, Gleich ist es in Bezug auf diejenigen, die ungläubig sind, ob du sie warnst oder nicht warnst; sie glauben nicht. “ Koran $2 / 6$

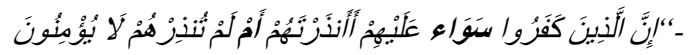

$\underline{\mathrm{d}-\text { Weder ...... noch }}$

Mit einer Negation wird immer das arabische Äquivalent für weder ... noch angezeigt. Wenn es mehrere Verben vorhanden ist,

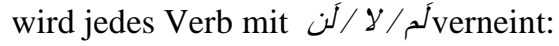

-Ich habe weder gegessen noch getrunken.

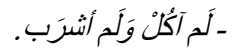

$$
\begin{aligned}
& \text { ـل آكُكْ وَلا أشرَبَ. }
\end{aligned}
$$

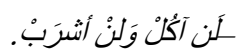

-Weder esse noch trinke ich.

-Ich werde weder essen noch trinken.

Die arabische Sprache verfügt über sein eigens System von Negationspartikeln. Während ${ }^{\top}$ zur Verneinung von Handlungen in der Vergangenheit dient, drückt $\gamma$ Handlungen sowohl in Gegenwart als auch in Zukunft aus, die Negationspartikel ${ }^{\prime}$ dagegen negiert Handlungen in der Zukunft.

Wenn mehrere Negationen aufeinander befolgt werden, wird zuerst mit وَلأ أو /ولَبِنَ:

-Er schreibt weder Briefe noch Bücher.

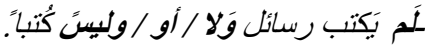

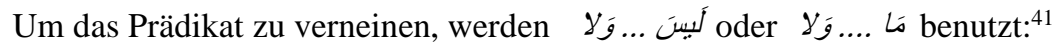

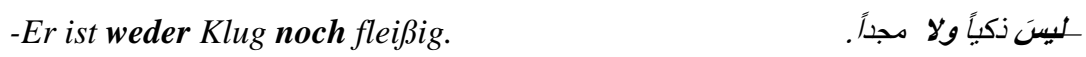

$\underline{\text { e-Nicht nur ...... sondern auch }}$

Vgl. Schulz, Eckehard, S. $167^{41}$ 
Es gibt im Arabischen mehrere Möglichkeiten zum Ausdruck der Bedeutung von der mehrteiligen Konjunktion nicht nur .... sondern auch:

-Nicht nur Bücher, sondern auch CDs.

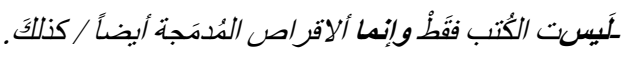

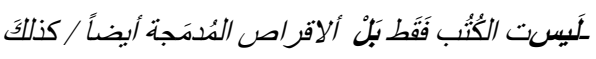

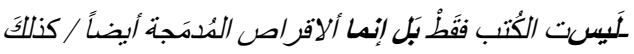

Außerdem kann noch ander Übersetzungsmöglichkeiten mit nicht nur .... sondern auch sowohl im heiligen Koran als auch in literarischen und grammatischen Texten gefunden werden.

\section{ERGEBNISSE UND BEWERTUNG}

\subsection{Unterschiede und Gemeinsamkeiten}

Es besteht kein Zweifel, dass der Vergleich der Grammatik der deutschen Sprache mit einer der östlichen Sprachen zu mehreren Unterschieden und einigen Gemeinsamkeiten führt, die mit beiden Sprachen übereinstimmen, von denen jede zu einer anderen Sprachfamilie gehört. Zum Produzieren eines sinnvollen Textes müssen Sätze durch Konjunktionen verknüpft werden. Die beiden Sprachen haben sowohl einteilige einfache Konjunktionen als auch mehrteilige korrespondierende Konjunktionen. In Bezug auf kopulative Konjunktionen handelt es sich im Deutschen um und, sowie, sowohl .... als auch, weder .... noch und nicht nur ...... sondern auch.

1- In den beiden Sprachen sind noch weitere Ausdrucksformem vorhanden, die ein formales Verknüpfungszeichen fehlt:

شir haben moderne wunderbare Häuser gesehen. شاهنا مَنازل حَديثة ور/ئعة

-Sehen Sie die Fülle des Wissens von Amr.

أنظُر الحى غزارة علم عمرو.

Die kopulativen Konjunktionen werden Sätze, Teilsätze oder kleinere Einheiten aneinander gereiht. Im Vordergrund steht die gehäufste Konjunktion der beiden Sprachen, nämlich und. Diese Konjunktion kann zwischen gehäuften Elementen stehen. Morpheme, Wörter, Wortgruppen, Phrasen, kommunikative Minimaleinheiten und Sätze können damit koordiniert werden.

2-Die Verbindungspartikeln الفَاء und im Arabischen ist als Äquivalente für die deutsche Konjunktion und zu betrachten. Also stimmt sowie und sowohl ..... als auch im Deutschen die arabische Verbindungspartikel اولواو überein.

3- in der arabischen Sprache enthält spezielle Bestimmungen, die sich nicht auf die deutsche Konjunktion und beziehen, nämlich in Verbindung mit der Bedingungspartikel إمّا , لكن und der Partikel und degationspartikel Vei Ordinalzahlen wie der 21. Tag - اليوم الحادي والعشرون und bei Schwur wie Bei Gott - والثئل

4-Außerdem wird im Arabischen das sogenannte واو المعية unterschieden, die eine Begleitung bezeichnet und der Akkusativ nach der Verbindungspartikel الو او eintritt:

Er hörte nicht auf mit dem Nil.

5- Sowohl im Deutschen als auch im Arabischen kann der Konjunktion und bei Verbindung gleicher Wörter zur Seigerung und Verstärkung eines Ausdrucks dienen:

Er läuft und läuft und läuft.

بركض ويرض ويركض

6-Im Gegensatz zum Arabischen steht im Deutschen bei einer Aufzählung die Konjunktion und vor dem letzten Glied, während es im Arabischen vor allen gleichrangigen Glieder steht: 
(IJRSSH) 2020, Vol. No. 10, Issue No. IV, Oct-Dec

Frühling, Sommer, Herbst und Winter.

الربيع و الصيف و الخريف والثتاء.

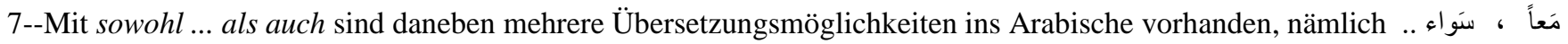
أم أم (أو) ، كُل

8--Hinsichtlich der mehrteilige Konjunktion weder ... noch unterscheidet man mehrere Ausdruckformen der

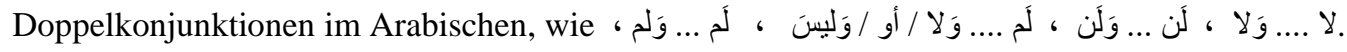

9-Die mehrteilige Konjunktion nicht nur ...... sondern auch kann im Arabischen mit ، لَّسن .. فَقَط و إنما أيضاً / كَذلكَ

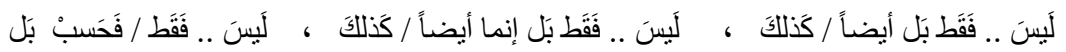

Übersetzt werden.

Im Folgenden ist eine Tabelle der kopulativen Konjunktionen im Deutschen und ihre entsprechende Übersetzungsmöglichkeiten im Arabischen:

\begin{tabular}{|c|c|c|c|c|}
\hline Und & Sowie & sowohl & nicht nur ..... Sondern auch & weder .... Noch \\
\hline 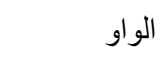 & 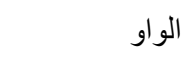 & 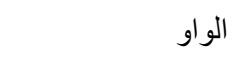 & ليس ... فقط و إنما أيضاً / كذللكَ & لَمْ ....... وَلَّم \\
\hline 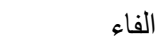 & أيضاً & سو اء ..... أم (أو) & ليس ... فقط بَل أيضاً / كَللك & لا....... و עل \\
\hline & وكنللك أيضاً & مel & ليس ... فقط بَل إنما أيضاً /كَللك & لن ........ وَأَن \\
\hline & & كُل & ليس ... فقط / فَحسب بَل أيضاً /كذللك & 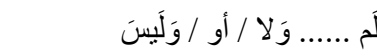 \\
\hline & & & لا .... فَقَط / فَحَسب و إنما أيضاً / كذللكَ & لا ...... و لا / أو / وليسن \\
\hline & & & & لَن ..... و لا / أو / وَلَيسنَ \\
\hline
\end{tabular}




\section{2 Übersetzungsschwierigkeiten}

Die Übersetung von Texten aus einer Sprache in eine andere ist keine leichte Aufgabe, da es Genauigkeit und Sorgfalt bei der Bereitstellung genauer Informationen erfordert. Es klingt schwieriger, wenn die Übersetzung eine Sprache aus einer europäischen und die andere einer östlichen Sprachfamilie betrifft, die sich von dieser unterscheidet, insbesondere in Bezug auf sowohl Sprachstile als auch grammatische Eigenschaften. Diese Arbeit beschäftigt sich mit der syntaktischen Verbindung zwischen Wörtern, Wortgruppen, oder Sätzen und zwar kopulative Konunktionen und deren Vergleich auf Arabisch. Solche Vergleiche führen zweifellos zu Unterschieden und Gemeinsamkeiten, die der Übersetzer in Betracht ziehen sollte.

Bei der Übertragung einer Satzkonstruktion von einer Sprache in die andere unterscheidet man folgendes:

1. Jede kopulstive Konjunktion sowohl einteilig als auch mehrteilig im Deutschen entspricht mindestens zwei oder mehr Übersetzungsmöglichkeiten im Arabischen.

2. Eine Konjunktion in einer Sprache ist nicht als Kriterium für die Vorhandensein der entsprechenden Konjunktion in die andere Sprache anzusehen.

3. Die arabische Sprache hat spezielle Bestimmungen mit الواو zum Ausdruck der verschiedenen Positionen, indem sie mit bestimmten Verbindungspartikeln zugordnet ist.

4. Bei der Übersetzung der Konjunktion und aus dem Arabischen ins Deutsche sollte berücksichtigt werden, ob diese mit der Präposition mit übereinstimmt.

5. Bei einer Aufzählung von mehreren Satzglieder oder gleichrangigen Adjektive sollte in beiden Sprachen die Position der Konjunktion und in Anschlag gebracht werden.

\section{LITERATURVERZEICHNIS}

1-Albert, Ruth: Vorlesungen, Probleme der Grammatikbeschreibung des Deutschen.

2-Autorenkollektiv: Deutsch Grammatik, 2009 Compact Verlag, München.

3-Autorenkollektiv: Duden Grammatik Band 4, Dudenverlag- Mannheim.Leipzig.Wien.Zürich, Bibiliogaphisches Institut AG, Mannheim 1984.

4-Autorenkollektiv: Duden, Die Grammatik, Band 4 Dudenverlag, Bibiliogaphisches Institut \& F.A. Brockhaus AG, Mannheim 2006.

5-Breindl, Eva: Handbuch der deutschen konnektoren, Februar 2006.

6.Brockelmann, Carl: Arabische Grammatik, Veb Verlag Enzyklopädie Leipzig, Druck: F. Ullmann KG, Zwickau - III/ 29/2.

7-Bußmann,Hadumod: Lexikon der Sprachwissenschaft,3. Stuttgart 2002 ,ISBN 3-520-45203-0.

8-Eisenberg, Peter: Grundriss der deutschen Grammatik, Band 2: Der Satz 1999, Verlag J. B. Metzler Stuttgart . Weimar.

9-Engel, Ulrich: Deutsche Grammatik, Julius Gross Verlag, 1996, D-69126 Heidelberg.

10-Götze, Lutz/ Hess- Lüttich, Ernest W. B.: Wahrig, Grammatik der deutschen Sprache, 2002 Wissen Media Verlag GmbH, Gütersloh/ München.

11-Helbig,G/Buscha,J: Deutsche Grammatik, Berlin u.a. 2001, Langenscheidt KG.ISBN 3-468-49493-9.

12-Helbig, Gerhard/ Buscha, Joachim: Leitfaden der Deutschen Grammatik, 2000 Langenscheidt KG, Berlin und München. 
13-Hentschel, Elke/ Wezdt, Harald: Handbuch der deutschen Grammatik, 2003 by Walter de Gruyter GmbH \& Co. KG, D10785 Berlin.

14-Heringer, Hans Jürgen: Grammatik und Stil, Cornelsen 1995.

15-Homberger, Dietrich: Grammatik für die Deutschunterricht, Ernst Klett Verlag, Leipzig 2001.

16-Hraishawi, Maher: Mehrteilige Konnektoren im Vergleich, Deutsch - Arabisch, Dissertation 2016.

17-Pash, Renate/ Brauße, Ursula und andere: Handbuch der deutsche Konnektoren, 2003 by Walter de Gruyter GmbH \& Co. KG, D-10785 Berlin.

18-Rudolf und Hoberg, Ursula: Der kleine Duden/ Deutsche Grammatik, 2., Dudenverlag, Mannheim 1997.

19-Schulz, Eckehard: Modernes Hocharabisch - Grammatik, Reichert Verlag Wiesbaden 2004.

20-Zifonun, Gisela/ Hoffman, Ludger und andere: Grammatik der deutschen Sprache 1997 by Walter de Gruyter GmbH \& Co., D-10785 Berlin

21- https://mawdoo3.com

22- https://qurblog.wordpress.com

$\underline{\text { Die Wörterbücher }}$

1-Arabisches Wörterbuch für die Schriftsprache der Gegenwart, Hans Wehr 1977.

2.Duden Band 2 - Das Stilwörterbuch, Bibliographisches Institut \& F.A. Brockhaus AG. Mannheim 2001.

3-https://studylibde.com/doc/1997153/kleines-glossar-zur-Einführung-in-die-sprachwissenschaft\%E2\%80\%9C

4-Schregle,G:Deutsch-Arabisches Wörterbuch 1977.

5-Wahrig Deutsches Wörterbuch, herausgegeben Renate Wahrig-Burfeind,2006.

6-Wahrig Synonymwörterbuch, 2002 Wissen Media Verlag GmbH, Gütersloh/ München. 


$$
\begin{aligned}
& \text { 1- القران الكريم وترجمة معانيه الى اللغة العربيةـ المدينة المنورة، رقم الإيداع 22/ 3909. } \\
& \text { 2- الجملة العربية و المعنى: الدكتورفاضل صالح السامر ائي } \\
& \text { 3-أوراق بحثنة في الترجمة: زينب عبد اللطيف صالح، دار المامون للترجمة والنشر ، بغداد } 2014 . \\
& \text { 4- -جامع الدروس العربية| مصطفى الغلاييني- الجزء الأول -الناشر شريف الأنصاري , بيروت } 1959 . \\
& \text { 5-شرح ابن عقيل لالفية ابن مالك في الصرف: الدكتور حسن عبد الجليل يوسف، مؤسسة المختار للنشر و التوزيع - القاهرة 2008. } \\
& \text { 6- علم المعاني: الدكتور بسيوني عب الفتاح فيود، مؤسسة المختار للنشر والتوزيع } 2010 . \\
& \text { 7-معاني النحو: الدكتور فاضل صالح السامر ائي، المجلد الأول ـ شركة العاتك للطباعة والنشر و التوزيع } 2003 . \\
& \text { 8- -معاني النحو: الدكتور فاضل صالح السامر ائي، المجلد الثاني ـ شركة العاتلك للطباعة والنشر والتوزيع } 2003 \\
& \text { 9-معاني النحو: الدكتور فاضل صالح السامر ائي، المجلد الثالث ـ شركة العاتك للطباعة والنشر و التوزيع } 2003 .
\end{aligned}
$$

